\title{
DE VOLTA AO BÁSICO: definições e processos de avaliação
}

\author{
BACK TO BASICS: definitions and \\ processes of assessments*
}

\author{
Maddalena Taras**
}

\begin{abstract}
Resumo
Num mundo educacional guiado pelo desempenho e pelos resultados, o conceito de avaliação formativa inspirou a comunidade da área com seu discurso e foco na aprendizagem e nos aprendizes. No entanto, várias controvérsias surgiram, destacando-se as nebulosidades e disparidades terminológicas entre os diversos setores de pesquisa e mesmo dentro de cada um deles (TARAS, 2007b, 2009). Perrenoud (1998), entre outros autores, assinala a importância de situar-se o discurso teórico e prático sobre a avaliação num contexto pedagógico mais amplo e no âmbito das teorias de aprendizagem. Taras (2005) argumenta que os conceitos de avaliação, incluindo-se a avaliação formativa, são mais bem entendidos e têm mais eficácia quando considerados numa estrutura mais abrangente e examinados segundo as suas relações, ou seja, quando se observam as relações entre avaliação somativa, avaliação formativa e autoavaliação. Este artigo, cujo propósito é examinar algumas definições de avaliação, apresenta primeiramente os conceitos básicos de avaliação, avaliação somativa, avaliação formativa, autoavaliação e feedback e em seguida os inter-relaciona. Os princípios implícitos nas definições determinam os parâmetros tanto dos processos quanto da prática como parte de uma estrutura e de uma sequência lógicas.
\end{abstract}

Palavras-chave: Avaliação. Avaliação formativa. Avaliação somativa.

\begin{abstract}
In a performance - and results - driven educational world the concept of formative assessment has inspired the educational community by its discourse and focus on learning and learners. However, a number of controversies have surfaced: primary among these are terminological opacities and disparities both within and across continents and sectors (TARAS 2007b, 2009). Among others, Perrenoud (1998) signals the importance of positioning theoretical and practical discourse on assessment within a wider pedagogic context and within theories of learning. Taras (2005) argues that concepts of assessment, including formative assessment, are best and more effectively understood firstly within the wider assessment framework and, secondly, within the relationships of summative, formative and self-assessment. This paper examines definitions of assessments. It begins with basic concepts of assessment, summative, formative, self-assessment and feedback and interrelates these. The principles inherent in definitions set the parameters of both processes and practice as part of a logical sequence and framework.
\end{abstract}

Keywords: Assessment. Formative assessment. Summative assessment.

\section{Introdução}

Ao explorar os princípios do processo de avaliação em qualquer contexto ou função, sejam estes preestabelecidos ou não, o presente artigo sustenta e desenvolve o discurso e o argumento de que, em todos os tipos de avaliação, entender o processo é mais importante do que observar as funções da avaliação, particularmente no caso da avaliação formativa (TARAS, 2005). Este texto não apresenta uma linha histórica do desenvolvimento das definições de avaliação formativa e avaliação somativa; tampouco constitui uma análise dos diferentes aspectos de avaliação vistos à luz de uma teoria geral da Pe-

\footnotetext{
*A versão original do artigo, em Língua Inglesa, encontra-se disponível na versão online da revista.

** Professora da University of Sunderland - Reino Unido. E-mail: maddalena.taras@sunderland.ac.uk. Tradução de Roberto Monteiro de Lima e revisão de Jefferson Mainardes.
} 
dagogia (cf. BLACK; WHITE, 2005; PERRENOUD, 1998), embora inevitavelmente considere a avaliação como parte do triunvirato ${ }^{1}$ avaliação-ensinoaprendizagem. $\mathrm{Na}$ verdade, este artigo defende a ideia de que não há desenvolvimento especializado sem avaliação (ATKINS et al., 1993).

\section{Teorias de aprendizagem}

De acordo com a teoria socioconstrutivista de aprendizagem, o indivíduo relaciona o significado com o meio e o contexto e o assimila reestruturando e reorganizando os conceitos e conhecimentos que possui (HAGER; HODKINSON, 2009; JAMES, 2006). Assim, o indivíduo é a um só tempo produto do contexto e um desafio direto ao mesmo, visto que reúne interpretações específicas e singulares. Do mesmo modo, a avaliação e a aprendizagem retomam a constante interação que o indivíduo desenvolve com a experiência coletiva e com as informações fundamentais que tiveram impacto nele e no contexto. Todos nós avaliamos constantemente a nossa situação, a nossa situação no coletivo e a situação do coletivo.

Portanto, cada indivíduo, embora aprenda e avalie num contexto socialmente construído, se diferencia dos outros em situações similares. Essa singularidade requer uma abordagem contínua dos significados, conceitos e ideias. Mesmo quando se trabalha com as mesmas definições, critérios e processos, a ocorrência de diferentes resultados de aprendizagem, avaliação e compreensão não pode ser considerada algo inusitado. Aceitar a infinita diversidade na educação é o primeiro passo importante rumo a uma relativa harmonização dos conceitos e definições, ideias, ideais e entendimentos. Isso, contudo, não elimina a necessidade de associações coerentes e lógicas no âmbito dessas experiências pessoais das "realidades" educacionais individuais e coletivas.

\section{Linguagem}

A linguagem não é neutra; os significados associados a diferentes palavras e termos não são neutros. A avaliação e os termos a ela relacionados são marcados por valores e emoções, do que decorre que fatores éticos e morais também estão envolvidos nas interpretações feitas pelo indivíduo e pela coletividade (FAIRCLOUGH, 1994; LAKOFF; JOHNSON,

\footnotetext{
1 Tradução literal do inglês triumvirate. Ambos os termos encontram sua origem na forma latina triumviratus, que designava a associação de três magistrados responsável pelo governo em Roma no período da República. Apesar de tríade nos ter parecido uma solução mais lógica e mais ao alcance do leitor, mantivemos a esfera política sugerida pela escolha da autora (N.T.).
}

1980; TARAS, 2007b). Do ponto de vista social e político, a "avaliação" é uma bomba em potencial, e nesses contextos a palavra é usada parcamente. A avaliação de artigos para revistas acadêmicas é conhecida como "revisão por pares". No Reino Unido, em 2008, o RAE (Research Assessment Exercise, ou Exercício de Avaliação da Pesquisa) ${ }^{2}$ causou polêmica pela sua potencial falta de transparência quando o feedback dos pareceristas foi destruído para proteger seus "julgamentos profissionais". Todos os anos há um furor quando os resultados dos exames nacionais são divulgados e dissecados pela mídia. No plano individual, histórias idiossincráticas e pessoais sempre acabam resultando em diferentes interpretações (COFFIELD; EDWARDS, 2009; HAGER; HODKINSON, 2009; TARAS, 2007b).

\section{Assessment ou evaluation ${ }^{3}$ ?}

Em virtude da globalização da pesquisa, a distinção entre assessment e evaluation no campo da Educação está se tornando cada vez mais complexa. Na literatura francófona (por exemplo, PERRENOUD, 1998) e nas línguas de base latina, em que inexiste uma distinção, geralmente se prefere usar evaluation.

No Reino Unido e na maior parte do mundo anglófono, a distinção costuma se dar da seguinte forma: o termo evaluation refere-se à macroesfera (por exemplo, universidade, curso, documentação de programas de ensino), ao passo que assessment diz respeito à microesfera (ou seja, às tarefas e trabalhos feitos pelos alunos). Essa distinção está essencialmente ligada ao contexto.

\footnotetext{
${ }^{2}$ No Reino Unido, o "Exercício de Avaliação da Pesquisa" (RAE Research Assessment Exercise) é um processo no qual cada universidade apresenta os melhores artigos de seus pesquisadores para serem avaliados. Isso geralmente ocorre a cada quatro anos e cada pesquisador apresenta, no máximo, quatro artigos. É com base nesses resultados que o governo recursos para pesquisa, de forma que um quantidade relativamente grande de recursos e prestígio estão em jogo. Os pareceristas que fizeram os julgamento da qualidade desses trabalhos no ano de 2008 ficaram tão preocupados com a integridade de suas avaliações fosse questionada que eles destruíram as anotações que explicavam as suas decisões. O Exercício de Avaliação de Pesquisa (RAE) passou a ser chamado de "Referencial de Excelência em Pesquisa" (REF -Research Excellence Framework), o que parece estar ligado a um certo medo do que está por trás da palavra avaliação.

${ }^{3}$ Mantivemos os termos ingleses assessment e evaluation nesta seção porque, como observa a própria autora, as línguas latinas não possuem mais de uma palavra para expressar satisfatoriamente a ideia contida em avaliação. Poderíamos, é verdade, tentar reproduzir a distinção do original substituindo evaluation por apreciação, por exemplo, mas entendemos que esse recurso seria limitado a ponto de induzir o leitor a interpretações equivocadas. Em contraponto à amplitude da palavra avaliação, as palavras apreciação e valoração parecem demasiadamente ligadas ao fator numérico; estimativa, por sua vez, está circunscrita à noção de valor aproximado (N.T.).
} 
Para o escopo deste trabalho, essa distinção é talvez superficial porque foca processos e princípios, almejando englobar contextos díspares. Assim, quer o nosso foco seja a avaliação de programas de ensino, como foi para Scriven (1967), quer tenhamos a nossa atenção voltada para o produto da avaliação em contextos complexos e com múltiplos critérios, como no caso de Sadler (1989), quer consideremos ainda a interação na sala de aula, como faz o trabalho de Black e William sobre a "avaliação para a aprendizagem"4, o que este artigo deseja argumentar e demonstrar é que um simples processo de avaliação pode ser usado para representar cada um desses três contextos bastante diversificados. Em outras palavras, o processo e os parâmetros básicos de avaliação podem ser considerados universais e tecnicamente similares em todos os contextos.

Outra distinção a ser considerada é aquela referente à avaliação implícita e à avaliação explícita. A primeira tende a abranger áreas de avaliação informal e ad $h o c^{5}$, isto é, refere-se à avaliação do trabalho em curso ou na interação da sala de aula, ao passo que a segunda tende a ser avaliação do produto, na qual os critérios e padrões são estabelecidos e compartilhados.

$\mathrm{Na}$ literatura, entende-se que os termos evaluation e assessment se sobrepõem e não são separados, o que torna a distinção difícil tanto na teoria quanto na prática (BLACK, 1998; CULLINGFORD, 1997; SCRIVEN, 1997). A natureza onipresente da avaliação foi há muito reconhecida, e as distinções destacadas nas linhas anteriores constituem essencialmente uma diferença de escopo e contexto e talvez reduzam o caráter comum do processo.

\footnotetext{
${ }^{4}$ A autora refere-se ao clássico e seminal trabalho de Paul Black e Dylan Wilian (1998), intitulado "Inside the Black Box: Raising Standards Through Classroom Assessment' (Phi Delta Kappan, October 1998). A partir do exame de uma vasta literatura, os autores oferecem fortes evidências que a avaliação formativa, quando implementada adequadamente, é um poderoso recurso para melhorar a aprendizagem dos estudantes. Por outro lado, a avaliação somativa, tais como os exames padronizados podem ter efeitos prejudiciais. A avaliação formativa ocorre quando os professores utilizam as informações obtidas por meio da avaliação para auxiliar os alunos a aprenderem mais e melhor. Assim, o propósito principal da avaliação é apoiar uma aprendizagem de melhor qualidade. Por essas razões, os autores utilizam a expressão "avaliação para a aprendizagem". Os autores mostraram também que avaliação formativa de alta qualidade era rara nas salas de aula. $\mathrm{Na}$ "avaliação para a aprendizagem", tanto o professor quanto o aluno usam as informações obtidas por meio da avaliação para modificar as atividades de ensino e aprendizagem. Informações complementares a respeito do artigo "Inside the Black Box" e do Grupo de Pesquisa sobre Avaliação liderado por Paul Black podem ser podem ser obtidas em: http://www.kcl.ac.uk/content/1/c4/73/57/ formative.pdf (Nota do revisor).

${ }^{5} \mathrm{O}$ fato de a autora ter preterido mais de uma vez qualquer adjetivo da sua língua em favor da expressão latina ad hoc deixa claro o quanto esta lhe é cara. Por esse motivo, não traduzimos a expressão e esclarecemos que ela designa algo utilizado para um fim específico. Nos âmbitos filosófico e científico, entende-se como elemento ad hoc qualquer recurso utilizado de maneira circunstancial, carecendo, portanto, de aplicação geral (N.T.).
}

A fim de coordenar e entender os princípios fundamentais da avaliação é útil incorporar todos os aspectos de 'evaluation' e 'assessment' num argumento único e coerente; isso abrangeria tanto o processo quanto o produto, tanto o informal quanto o formal.

\section{A definição de avaliação (assessment)}

O termo avaliação geralmente se refere a julgamento e constitui um processo que permeia grande parte das nossas vidas. No âmbito educacional, esse processo também se dá em todos os níveis e contextos, e muitos nomes the têm sido conferidos. Entretanto, talvez seja válido lembrar que avaliação é avaliação e que tudo pode ser e é julgado.

A definição transcrita a seguir descreve e faz referência ao processo de avaliação e explica como se chega ao julgamento. Conforme foi observado, esse julgamento pode ser tanto do processo quanto do produto, explícito ou implícito, formal ou informal, ou de qualquer ponto desse contínuo.

\begin{abstract}
A avaliação é uma atividade metodológica essencialmente similar em qualquer contexto: não importa se estamos avaliando máquinas de café ou máquinas de ensinar ${ }^{6}$, planos para uma casa ou planos para um curso. Trata-se de uma atividade que consiste simplesmente na junção e combinação de dados de desempenho com um conjunto ponderado de escalas de objetivos para gerar listas comparativas ou numéricas, com base (a) nos instrumentos de coleta de dados, (b) nas ponderações e (c) na seleção dos objetivos. (SCRIVEN, 1967, p. 40).
\end{abstract}

Scriven clarifica que esses são princípios universais e esse artigo ilustra esse fato com o exemplo da avaliação de programas.

\section{Funções da avaliação somativa e da avalia- ção formativa}

Nos últimos trinta anos, as avaliações somativa e formativa passaram a ser definidas e caracterizadas cada vez mais pelas suas funções. As funções ou papéis (termos usados intercambiavelmente neste artigo) são o uso ou propósito ao qual se destina a avaliação, e isso pode ser decidido antes, durante

\footnotetext{
${ }^{6}$ As máquinas de ensinar (teaching machines) eram aparelhos destinados a apresentar de maneira automática um conteúdo didático cuidadosamente planejado. Enquanto o aluno não encontrasse a solução da questão ou exercício proposto, ele não poderia seguir em frente. Skinner foi um dos grandes propagadores desses aparelhos, e de fato é notório o conceito comportamentalista por trás dos mesmos: a aprendizagem se daria por meio do reforço das respostas corretas, propiciado pela significativa repetição de exercícios semelhantes (N.T.).
} 
ou após a avaliação. No ensino superior, a distinção parece ter sido tratada de modo pragmático, já que todas as avaliações encontram-se sob controle institucional e, portanto, não estão em conflito com agentes externos (TARAS, 2008c). Brown e Knight (1994) representam essa tendência: "Há muitas misturas de propósitos, o que reflete os múltiplos públicos da avaliação e a grande quantidade de alternativas para se avaliar o ensino. 'Formativa' e 'somativa' são rótulos úteis, mas não mais do que isso" (BROWN; KNIGHT, 1994, p. 15-16).

No ensino obrigatório, no qual os exames são controlados por agências externos, o conflito é mais evidente (Black; William, 1998; Sebatane, 1998). O trabalho liderado por Black e William e grande parte da pesquisa de Avaliação para a Aprendizagem no ensino obrigatório baseiam-se nessa distinção entre avaliação somativa e avaliação formativa, onde a avaliação somativa refere-se à validação externa dos exames e a avaliação formativa como sendo aquele que oferece elementos para o feedback na sala de aula. A ênfase na distinção e separação desses dois tipos de avaliação tem sido apontada por Biggs (1998) como uma deficiência do inspirador artigo de Black e William (1998). ${ }^{7}$

Esse foco nas funções da avaliação foi iniciado em parte por Bloom et al. (1971), que investigaram as consequências da avaliação e concluíram que elas são tão importantes quanto os processos ou propósitos. Embora reconheça a importância fundamental das consequências, este artigo não foca esse aspecto, limitando-se aos processos de avaliação.

\section{Avaliação somativa}

A avaliação somativa é em geral igualada às provas ou testes finais. No Ensino Superior, a prática de dar um feedback (ou retorno) ${ }^{8}$ sobre um trabalho do aluno mostra que a avaliação somativa e a avaliação formativa foram utilizadas em conjunto e que se apoiaram para o mesmo fim, qual seja, auxiliar a aprendizagem dos alunos. Isso é relatado na literatura que em geral não tece considerações negativas a respeito da avaliação somativa (BIGGS, 1998; BROWN; KNIGHT, 1994). No discurso da "avaliação para a aprendizagem", esse tipo de avaliação é geralmente depreciado e tomado como o propulsor dos aspectos negativos e destrutivos da educação

\footnotetext{
${ }^{7}$ A autora refere-se Paul Black e Dylan Wilian (1998), intitulado "Inside the Black Box: Raising Standards Through Classroom Assessment" (Phi Delta Kappan, October 1998), citado a nota 3. (N. do revisor)

${ }^{8}$ Apesar de não impor dificuldades de entendimento, a palavra feedback é de difícil tradução para o português. As variadas soluções, como retroalimentação, parecer, resposta e retorno, entre outras, dependem, sobretudo do contexto. Por exemplo, retroalimentação é aplicável a máquinas, mas jamais à relação professor-aluno. (N.T.).
}

não comprometida com o apoio à aprendizagem (TARAS, 2007b, 2008c). Nas palavras de Broadfoot (2002, 2007, 2008), a avaliação somativa é "um monstro de Frankenstein". Os subtítulos constantes do seu artigo de 2008 não deixam dúvida quanto à sua visão cáustica sobre as "funções" da avaliação somativa e quanto ao efeito desta nas vidas dos aprendizes e da comunidade educacional ${ }^{9}$. Contudo, a avaliação somativa e os exames externos não são sempre negativos, pois tradicionalmente o sucesso nas provas tem sido uma rota para um futuro melhor para os alunos. O problema reside nas implicações e práticas distorcidas, as quais datam de milênios (BROADFOOT, 2007; STOBART, 2008).

\section{Funções e processos de avaliação}

Uma questão importante para este artigo é: como as funções da avaliação se ligam ao processo de avaliação? Uma dada função altera ou influencia o processo de alguma maneira? A resposta é não: o processo de avaliação não é afetado pelas potenciais funções desta. Portanto, o processo e as funções são autônomos.

Por outro lado, o foco nas funções tem ofuscado a análise dos processos, tornando difícil controlar o aspecto vital de entender o processo e garantir que este seja transparente e ético. Scriven (1967) já havia alertado sobre essa ocorrência. Desde então, um segundo problema parece ter surgido: a separação entre processos de avaliação somativa e avaliação formativa, um reflexo das recomendações da literatura em apoio ao trabalho com funções da avaliação (TARAS, 2008c, 2009). Essa situação requer a duplicação das avaliações somativa e formativa para que as diferentes funções sejam observadas (BLACK, 2003; WILLIAM, 2000). Taras $(2005,2009)$ demonstra que essa duplicação é desnecessária, demorada e confusa tanto para os professores quanto para os alunos.

\section{Funções}

As funções são consideradas um problema neste texto por dois motivos: primeiro, dominam a literatura recente; segundo, são responsáveis por fazer os especialistas em Educação perderem a visão sobre os processos de avaliação e sobre a neutralidade essencial da avaliação em si.

\footnotetext{
${ }^{9} \mathrm{~A}$ autora refere-se ao seguinte ao seguinte texto: Broadfoot, $\mathrm{P}$. (2008). Assessment for learners: Assessment literacy and the development of learning power. In: Havnes, A.; McDowell, L. (Eds) (2008). Balancing Dilemmas in Assessment and Learning in Contemporary Education. New York/London: Routledge, p. 213-224.
} 
O primeiro fato - o domínio na literatura - não é necessariamente algo ruim, uma vez que pode contribuir para nos lembrar de que, ao longo da história, a avaliação foi usada injustamente e chegou inclusive a arruinar muitas vidas (STOBART, 2008). Então, a pergunta que cabe é: podemos controlar o modo como a avaliação é utilizada? A resposta é não. Ainda que priorizássemos funções positivas e éticas, como o auxílio à aprendizagem, não poderíamos assegurar que os resultados do processo de avaliação seriam usados como pretendido (TARAS, 2005, 2007c). Assim, focar as funções não irá contribuir para garantir que a avaliação seja usada com ética. No que tange ao segundo fato, as consequências da perda de visão sobre o processo de avaliação são muito sérias: a não monitoração dessa perda significa efetivamente que não estamos garantindo nem que a avaliação seja realizada apropriadamente nem que os resultados sejam relatados de modo transparente. Esse cenário, por sua vez, contribui para tornar a neutralidade essencial da avaliação (terceiro ponto) ainda menos neutra. Há ainda sérias implicações para a confiabilidade e validade da avaliação, mas a discussão desse tópico está além do escopo deste artigo.

Seria possível argumentar que esse discurso está criando uma tempestade em copo d'água. No entanto, se esse copo é a avaliação de um indivíduo, pode ter sérias implicações para o seu futuro. Além disso, pode-se argumentar que o foco nas funções formativas não irá infringir a integridade, validade ou confiabilidade do trabalho formativo. Conforme foi mencionado, o problema é que em geral as avaliações têm múltiplas funções e o que começa como um exercício de aprendizado informal, implícito e provavelmente não rigoroso pode ser usado para decisões vitais. Como resultado, os julgamentos finais e importantes podem decorrer de avaliações informais e ad hoc.

Para se evitar isso, é preciso que as avaliações tenham o rigor e o cuidado dispensados aos trabalhos somativos - mas com a intenção de auxiliar o ensino-aprendizagem - e os atributos positivos geralmente associados à avaliação formativa. Esse objetivo estará ao nosso alcance se focarmos os processos de avaliação em lugar das suas funções.

\section{O processo de avaliação}

O processo de avaliação está implícito nesta definição, que é repetida por conveniência:

Trata-se de uma atividade que consiste simplesmente na junção e combinação de dados de de- sempenho com um conjunto ponderado de escalas de objetivos para gerar listas comparativas ou numéricas, com base (a) nos instrumentos de coleta de dados, (b) nas ponderações e (c) na seleção dos objetivos (SCRIVEN, 1967, p. 40).

Assim, os parâmetros escolhidos, com justificativas, são: os instrumentos de coleta de dados, (b) as ponderações e (c) a seleção de objetivos. A avaliação é um processo complexo no qual todos os elementos usados para o julgamento estão em constante interação. Consequentemente, o resultado pode ser comparado a um padrão ou a um número de uma escala padronizada.

A avaliação somativa oferece informações que Sadler (1989) chama de "conhecimento dos resultados". Essas informações podem estar na forma de um boletim ou podem ser "comparativas". Neste último caso, o adjetivo comparativo alude ao deficit entre o desempenho perfeito ou ideal e o desempenho que está sendo avaliado. Portanto, a definição de Scriven antecipa parte da definição de feedback de Ramaprasad, assinalando uma "lacuna" a ser preenchida (1983).

\section{Feedback e avaliação formativa}

A definição de avaliação formativa é talvez a mais controversa e variada de todas as definições de avaliação já apresentadas, assim como a sua relação com a avaliação somativa e a autoavaliação. Como conceito, e à semelhança do seu parceiro próximo, o feedback, a avaliação formativa não é novidade: foca significados, técnicas e procedimentos a fim de auxiliar a aprendizagem por intermédio do feedback. Desse modo, o feedback é um aspecto crucial da avaliação formativa. Enquanto a avaliação somativa produz o feedback, a avaliação formativa precisa usá-lo.

Sadler adota a definição de Ramaprasad na sua teoria da avaliação formativa. Essa definição demonstra que o feedback, ao contrário do conhecimento dos resultados, é um processo complexo que exige a participação ativa dos aprendizes para lhes estimular o desenvolvimento. Exige também um entendimento do contexto de avaliação e dos parâmetros, bem como que os aprendizes compreendam a sua posição e a sua própria base de conhecimento nesse contexto:

[O fedback] exige conhecimento do padrão ou do objetivo, habilidades para fazer comparações com base em vários critérios e o desenvolvimento de estratégias e significados para reduzir a discrepância entre o que é produzido e o que é almejado (SADLER, 1989, p. 142). 
Como, de acordo com essa definição, o feedback é o primeiro passo necessário para a avaliação formativa, ele poderia ser denominado feedback formativo. No entanto, se olharmos a definição de Ramaprasad, entenderemos que se trata mais do que simplesmente um estímulo ao desenvolvimento: "O feedback é uma informação referente à lacuna entre o nível real de um sistema de parâmetros e o seu nível de referência, a qual é usada para alterar a lacuna de alguma forma" (RAMAPRASAD, 1983, p. 4, grifos nossos).

Na verdade, a definição de Sadler de avaliação formativa não é muito diferente da definição de feedback apresentada por Ramaprasad (ou feedback formativo).

\begin{abstract}
A avaliação formativa está relacionada ao modo como os julgamentos sobre a qualidade das respostas dos alunos (desempenho, trabalhos) podem ser usados para moldar e aprimorar a competência destes por meio de um rompimento com o caráter aleatório e a ineficiência da aprendizagem baseada na tentativa e erro (SADLER, 1989, p. 120, grifos nossos).
\end{abstract}

Como observa Taras (2005), o verbo modal pode mostra que, quando os julgamentos são usados, se trata de avaliação formativa. Se, ao contrário, não se faz nenhum julgamento, resta apenas o julgamento representado pela avaliação somativa. Evidentemente, isso nos leva a examinar a relação entre avaliação somativa e avaliação formativa.

\section{Relação entre avaliação somativa, avaliação formativa e feedback}

Do exposto até agora, conclui-se que fazer um julgamento com base em parâmetros específicos é uma avaliação, ou avaliação somativa, até então. Essa avaliação produz o feedback, que pode permanecer um julgamento implícito na mente do indivíduo, do contrário qualquer manifestação ou comunicação desse julgamento fornecerá informações. Segundo as definições de avaliação propostas neste texto, os parâmetros para se fazer um julgamento - os quais são os critérios, os modelos e os objetivos - são usados também para medir o deficit do ideal. As informações produzidas fornecem o feedback necessário para a melhoria do trabalho. O uso do feedback formativo pelo aprendiz resulta na avaliação formativa e aproxima o trabalho do ideal.

Taras (2005) representa essa relação na equação:

AS + feedback = AF (Avaliação Somativa + feedback $=$ Avaliação Formativa).
Mais precisamente, uma avaliação somativa produz feedback cujos resultados foram utilizados na avaliação formativa:

$$
\begin{aligned}
& \text { AS } \rightarrow \text { feedback } \\
& \text { Uso do feedback = Avaliação Formativa }
\end{aligned}
$$

Esse esquema, longe de apresentar a avaliação somativa e a avaliação formativa como itens separados, mostra que os dois tipos de avaliação são inseparáveis e que a avaliação somativa é o ponto de partida necessário para qualquer avaliação (TARAS, 2009).

Portanto, a avaliação somativa precisa vir em primeiro lugar: é necessário avaliar a qualidade do trabalho antes de dar o feedback ao aluno. O feedback não vem do nada: é o exame do trabaIho com critérios e modelos implícitos e explícitos que produz julgamentos. A diferença entre a avaliação somativa e a avaliação formativa reside no fato de que a última é usada pelo aprendiz para atualizar e aperfeiçoar o trabalho (ou, no mínimo, para entender o que precisaria ser feito e como). A avaliação somativa, por sua vez, não exclui o feedback (ou "conhecimento dos resultados"), e até mesmo uma nota ou reação física é capaz de fornecer informações, ainda que mínimas. No Ensino Superior, via de regra, os trabalhos que recebem nota são a principal fonte de fedback (TARAS, 2006).

Se, por um lado, o uso do feedback é avaliação formativa, por outro, a avaliação somativa também pode produzir - e em geral o faz - feedback passível de ser utilizado. $\mathrm{Na}$ avaliação formativa, o seu uso é obrigatório, ao contrário do que ocorre na avaliação somativa. Por ser um processo constante e universal e poder ser descrita por infinitos meios, grande parte da avaliação é implícita, automática e dada como certa. Talvez nós estejamos inclinados a esquecer a premissa básica e óbvia do processo. Coffield e Edward (2009) ilustram como a falta de comprometimento com as premissas básicas dos "bons" princípios de avaliação leva a práticas e pesquisas inferiores - e eu incluiria "teoria".

\section{Relação entre avaliação somativa, avaliação formativa e autoavaliação}

Examinamos as relações entre os processos de avaliação somativa e avaliação formativa. Nesta seção, iremos explorar como a autoavaliação se relaciona a tais processos. A literatura sobre autoavaliação a considera como avaliação formativa, além de tomá-la como o aspecto mais importante do auxílio à aprendizagem (BLACK et al., 2003; BOUD, 1995; COWAN, 2006). A literatura discutida neste artigo parece apresentar as mesmas convicções. 
Contudo, tanto Scriven (1967) quanto Sadler (1989) demonstram implicitamente que a autoavaliação é na verdade um processo somativo.

A menos que seja totalmente ignorante quanto às deficiências de outrem ao julgar-lhe um trabalho, ele [sic] está supostamente empenhado em comprovar a eficiência do trabalho enquanto este é desenvolvido e, ao fazer isso, obtém um feedback a partir do qual produz novas revisões; obviamente, isso é avaliação formativa (SCRIVEN, 1967, p. 43).

Segundo essa citação, a avaliação formativa usa feedback produzido pela avaliação somativa. Entretanto, como a mesma pessoa fornece o feedback e o utiliza, o que se tem é uma autoavaliação. Com base nisso, podemos entender que a produção de feedback pelo próprio indivíduo é um processo somativo, já que o indivíduo não é obrigado a usar esse feedback. De fato, todos nós devemos reconhecer que não extraímos o melhor de nossas habilidades em vista de tempo e restrições logísticas (TARAS, 2003).

Por outro lado, seria possível argumentar que qualquer processo de produção envolve feedback em curso e ad hoc pela pessoa em questão, o qual é subsequentemente incorporado em nível cognitivo juntamente com o trabalho. De fato, o uso obrigatório apregoado por Sadler da autoavaliação como parte integral da avaliação formativa idealiza esse processo. Taras (2009) considera que a autoavaliação é, do ponto de vista técnico e teórico, um processo somativo e que qualquer feedback produzido pelos aprendizes para si próprios precisaria ser utilizado para que pudesse ser considerado formativo. A indagação quanto à possibilidade de esse uso ser demonstrável em contexto escolar negligenciaria o processamento mental, que é onde acontece a verdadeira aprendizagem e avaliação.

\section{Implicações dos processos e funções da avaliação}

Este artigo demonstrou que, para se entender e focar o processo de avaliação é necessário favorecer e prover uma prática de qualidade. A avaliação é demasiadamente importante e possui consequências muito sérias para depender de processamento implícito e ad hoc. Todos temos consciência dos aspectos inerentemente subjetivos de todo e qualquer julgamento, mas tornar os parâmetros, processos e produtos explícitos e transparentes é de grande valia para produzir avaliações justas e éticas para todos os envolvidos. Ademais, como explicitado no início deste trabalho, a avaliação é uma parte integral e necessária da aprendizagem. Uma vez que a aprendizagem depende do contexto e é um produto do mesmo, a avaliação está igualmente ligada ao con- texto e é ainda um entendimento do que precisa ser negociado entre os protagonistas. Por fim, é essencial destacar que o feedback só se constitui como tal se for compreendido, aceito e integrado pelos aprendizes aos seus futuros trabalhos.

\section{Conclusão}

Os conceitos mencionados têm importantes implicações para a aprendizagem, o ensino e a avaliação. Os três, aliás, são interdependentes e exigem cooperação entre todos os envolvidos. Em qualquer contexto - seja na revisão de programas de ensino ou artigos por pares, seja numa entrevista de emprego, seja numa sala de aula, onde o professor e os alunos avaliam o próprio trabalho e o trabalho do outro ou as suas próprias ideias e ideais graças à interação propiciada pelo ambiente -, o processo de avaliação é basicamente o mesmo. Compartilhar parâmetros, práticas e contextos será fundamental para se chegar a um entendimento justo capaz de reduzir as injustiças que há muito tem assolado os processos de avaliação (BROADFOOT, 2008; STOBART, 2008). Dada a grande importância de toda e qualquer avaliação, seja somativa, seja formativa, precisamos ter coragem e conhecimento para sermos explícitos e transparentes. Este artigo mostra que distinguir as funções da avaliação somativa e da avaliação formativa não é necessariamente a resposta se o processo é ofuscado e ignorado.

\section{Referências}

ATKINS, M. J.; BEATTIE, J.; DOCKRELL, W. B. Assessment issues in higher education. Sheffield: Employment Department, 1993.

BIGGS, J. Assessment and classroom learning: a role for summative assessment? Assessment in education: principles, policy and practice, v. 5 , n. 1, p. 103-110, 1998.

BLACK, P.; WILIAM, D. Assessment and classroom learning. Assessment in education: principles, policy and practice, v. 5, n. 1, p. 7-74, 1998.

BLACK, P. Testing: friend or foe? - Theory and practice of assessment and testing. London: Falmer Press, 1998.

BLACK, P. (with the King's College London Assessment for Learning Group HARRISON, C.; LEE, C.; MARSHALL, B.; WILIAMS, D.). Formative and summative assessment: can they serve learning together? Paper presented at AERA Chicago 23 April 2003. SIG Classroom Assessment Meeting, Chicago, 2003.

BLACK, P.; HARRISON, C.; LEE, C.; MARSHALL, B.; WILIAM, D. Assessment for learning: putting it into practice. Maidenhead: Open University Press, 2003.

BLACK, P.; WILIAM, D. Lessons from around the world: how policies, politicsand culturesconstrainandaffordassessment 
practices. The Curriculum Journal, v. 16, n. 2, p. 249261, 2005.

BLOOM B. S.; HASTINGS, J. T.; MADAUS, G. F. (Eds.). Handbook on the formative and summative evaluation of student learning. New York: McGraw-Hill, 1971.

BOUD, D. J. Enhancing learning through self assessment. London: Kogan Page, 1995.

BROADFOOT, P. Editorial: Beware the consequences of assessment! Assessment in education: principles, policy and practice, v. 9, n. 3, p. 285-288, 2002.

BROADFOOT, P. An introduction to assessment. New York, London: Continuum, 2007.

BROADFOOT, P. Assessment for learners: assessment literacy and the development of learning power. In: HAVNES, A.; MCDOWELL, L. (Eds.). Balancing dilemmas in assessment and learning in contemporary education. New York, London: Routledge, 2008. p. 213-224.

BROWN, S.; KNIGHT, P. Assessing learners in higher education. London: Kogan Page, 1994.

COFFIELD, F.; EDWARDS, S. Rolling out 'good', 'best' and 'excellent' practice. What next? Perfect practice? British Educational Research Journal, v. 35, n. 3, p. 371-390, 2009.

COWAN, J. On becoming an innovative university teacher: reflection in action. Oxford: Oxford University Press, 2006.

CULLINGFORD, C. (Ed.). Assessment versus evaluation. London: Cassell, 1997.

FAIRCLOUGH, N. Discourse and social change. Cambridge: Polity Press/Blackwell, 1994.

GARDNER, J. (Ed.). Assessment and learning. London: Sage, 2006.

HAGER, P.; HODKINSON, P. Moving beyond the metaphor of transfer of learning. British Educational Research Journal, v. 35, n. 4, p. 619-638, 2009.

JAMES, M. Assessment, teaching and theories of learning. In: GARDNER, J. (Ed.). Assessment and learning. London: Sage, 2006. p. 47-60.

LAKOFF, G.; JOHNSON, M. Metaphors we live by. Chicago: University of Chicago Press, 1980.

LAKOFF, G.; JOHNSON, M. The contemporary theory of metaphor. In: ORTONY, A. (Ed.). Metaphor and thought. 2. ed. Cambridge: Cambridge University Press, 2002. p. 202-251.

PERRENOUD, P. From formative evaluation to a controlled regulation of learning. Assessment in Education: principles, policy and practice, v. 5, n. 1, p. 85-103, 1998

RAMAPRASAD, A. On the definition of feedback. Behavioural Science, v. 28, p. 4-13, 1983.

SADLER, D. R. Formative assessment and the design of instructional systems. Instructional Science, v. 18, p. 145-165, 1989.

SCRIVEN, M. The methodology of evaluation. In: TYLER, R.; GAGNE, R.; SCRIVEN, M. Perspectives on curriculum evaluation. AERA Monograph Series - Curriculum Evaluation, Chicago: Rand McNally and Co., 1967. p. 3883.

SEBATANE, E. M. Assessment and classroom learning: a response to Black and Wiliam. Assessment in education: principles, policy and practice, v. 5, n. 1, p. 123-130, 1998.

STOBART, G. Testing times: the uses and abuses of assessment. New York/London: Routledge, 2008.

TARAS, M. To feedback or not to feedback in student selfassessment. Assessment and Evaluation in Higher Education, v. 28, n. 5, p. 549-565, 2003.

TARAS, M. Assessment - Summative and Formative - some theoretical reflections. British Journal of Educational Studies, v. 53, n. 3, p. 466-478, 2005.

TARAS, M. Do unto others or not? Lecturers use expert feedback on research articles, why not likewise undergraduates on assessed work? Assessment and Evaluation in Higher Education, v. 31, n. 3, p. 363-375, 2006.

TARAS, M. Terminal terminology: the language of assessment. In: REISS, M.; HAYES, R.; ATKINSON, A. (Eds.). Marginality and difference in education and beyond. Staffordshire: Trentham Books, 2007b. p. 52-67.

TARAS, M. Assessment for Learning: understanding theory to improve practice. Journal of Further and higher education, v. 31, n. 4, p. 363-371, 2007c.

TARAS, M. Assessment: sectarian divisions of terminology and concepts. Journal of Further and Higher Education, v. 32 , n. 4 , p. $389-397,2008$ c.

TARAS, M. Summative Assessment: the missing link for formative assessment. Journal of Further and Higher Education, v. 33, n. 1, p. 57-69, 2009.

WILIAM, D. Integrating summative and formative functions of assessment. Keynote address to the European Association for Educational Assessment. Prague: Czech Republic, 2000.

Recebido em 05/09/2009 Aceito em 11/03/2010 